\title{
Removal of Image Blurring and Mix Noises Using Gaussian Mixture and Variation Models
}

\author{
Vipul Goel \\ Dept. of Electronics Engineering, Harcourt Butler Technical University, Kanpur, 208002, India \\ Email: vipgo1994@gmail.com \\ Dr. Krishna Raj \\ Dept. of Electronics Engineering, Harcourt Butler Technical University, Kanpur, 208002, India \\ Email:kraj_biet@yahoo.com
}

Received: 08 September 2017; Accepted: 22 September 2017; Published: 08 January 2018

\begin{abstract}
For the past recent decades, image denoising has been analyzed in many fields such as computer vision, statistical signal and image processing. It facilitates an appropriate base for the analysis of natural image models and signal separation algorithms. Moreover, it also turns into an essential part to the digital image acquiring systems to improve qualities of an image. These two directions are vital and will be examined in this work. Noise and Blurring of images are two degrading factors and when an image is corrupted with both blurring and mixed noises, de-noising and de-blurring of the image is very difficult. In this paper, Gauss-Total Variation model (G-TV model) and Gaussian Mixture-Total Variation Model (GM-TV Model) are discussed and results are presented. It is shown that blurring of the image is completely removed using G-TV model; however, image corrupted with blurring and mixed noise can be recovered with GM-TV model.
\end{abstract}

Index Terms-G-TV, GM-TV, Blurring and Noise.

\section{INTRODUCTION}

The field of digital image processing alludes to the use of computer algorithms to extract helpful data from digital images [1-4]. The whole procedure of image processing may be divided into three prime stages:

1) Image acquisition: Converting 3-D visual information into 2-D digital form appropriate for storage, processing and transmission.

2) Processing: Enhancing quality of an image by enhancement, restoration, etc.

3) Analysis: Drawing out image features; quantifying shapes and then recognition.

In image acquisition stage, input is an actual 3-D scene while output is a corresponding digital image. On the other hand, both input and output are digital images at second stage of processing, where the output is an enhanced form of the input. In the last stage, input is still a digital image but the output is description of its contents. Fig. 1 illustrates a block diagram.

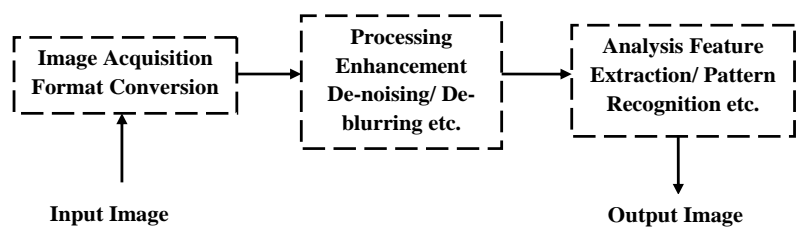

Fig.1. Image processing and noise filtering

\section{A. Noise}

Noise in an image is the irregular variation of color or brightness of the image created by the sensor and circuitry of a scanner or digital camera [5-13]. Image noise is taken as an unwanted by-product of captured image. The sorts of noises are as given below:

1) Gaussian noise (Amplifier noise): The standard model of amplifier noise is additive. Gaussian noise is independent at every pixel and also independent of the signal intensity.

2) Salt-and-pepper noise: An image comprising saltand-pepper noise consists of dark pixels in bright regions and bright pixels in dark regions.

3) Speckle noise: Speckle noise is a granular noise that inherently exists and corrupts the quality RADAR images. SAR is produced by unified processing of backscattered signals from numerous distributed targets.

\section{B. Gaussian Blur}

Gaussian Blur is that pixel weights aren't equal and they decrease from kernel center to edges. The effect of Gaussian Blur is a filter which de-focuses an image. The blurring is dense in the center while at the edge is feathers [12-13].

Frequently, digital cameras have very little noise in their pictures. Here we will illustrate an approach to dispose of that noise by making use of the selective Gaussian blur filter [14-16]. The fundamental idea behind specific Gaussian blur is that the photo areas with contrast below a certain threshold get blurred.

The composition of paper is as follows: In section II, various restoration filters are discussed. In Related Work Gauss-Total Variation model (G-TV model) is presented 
in section III. In section IV, Gaussian Mixture-Total Variation Model (GM-TV model) is presented. In section $\mathrm{V}$ of the paper simulation results are discussed. Finally, section VI of the paper concludes the work.

\section{VARIOUS IMAGE RESTORATION FILTERS}

Restoration of image or Denoising is the method of getting the original image from the corrupted image given the knowledge of the degrading factors [12-17]. It is utilized to evacuate noise from the degraded image without affecting and maintaining the edges with other details.

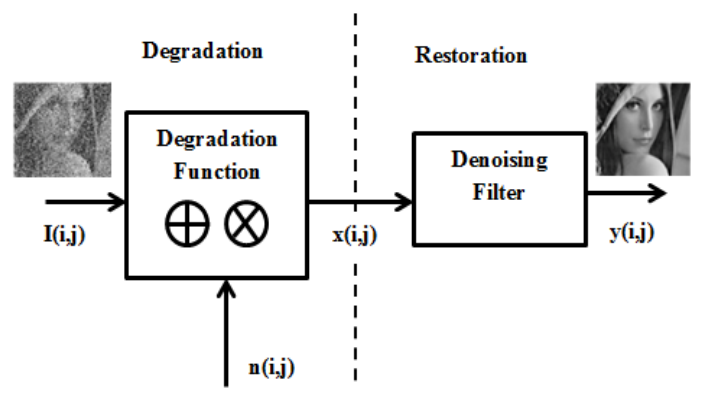

Fig.2. Image Degradation and Restoration process

In Fig. 2, image degradation and restoration process is shown. $O(i, j)$ is an input object $n(i, j)$ is degrading term (may include noise, blurring or both) so $x(i, j)$ is

$$
x(i, j)=O(i, j)+n(i, j)
$$

or

$$
\begin{gathered}
x(i, j)=O(i, j) \times n(i, j) \\
y(i, j)=H[x(i, j)]
\end{gathered}
$$

$H$ is filter operator.

In simplified form,

$$
y(t)=\int_{-\infty}^{\infty} x(\tau) h(t-\tau) d \tau
$$

where $x(t)$ is input image, $h(t)$ is filter impulse function and $y(t)$ is the output image.

\section{GAuss-TOTAL VARIATION MODEL (G-TV MODEL)}

A new interpretation of the ROF model is developed in this section that is based on statistical approaches. In the following, we consider that the noise intensity $n(x)$ or $(k * f)(x)+n(x)$ is a random variable and all these random variables are independent and identically- distributed (i.i.d.) as a Gaussian distribution $N\left(0, \sigma^{2}\right)$, i.e.,

$$
\begin{gathered}
g(x)=\left(k^{*} f\right)(x)+n(x) \\
p\left(\left(k^{*} f\right)(x)-g(x) / \sigma^{2}\right)=\frac{1}{\sqrt{2 \pi \sigma^{2}}} \exp -\left\{\frac{\left|\left(k^{*} f\right)(x)-g(x)\right|^{2}}{2 \sigma^{2}}\right\} \\
L\left(f, \sigma^{2}\right)=\prod_{x \in \Omega} \frac{1}{\sqrt{2 \pi \sigma^{2}}} \exp -\left\{\frac{\left|\left(k^{*} f\right)(x)-g(x)\right|^{2}}{2 \sigma^{2}}\right\}
\end{gathered}
$$

Minimizing log-likelihood function

$$
\begin{aligned}
E_{1}\left(f, \sigma^{2}\right)= & \frac{1}{2} \int_{\Omega}\left\{\frac{\left|\left(k^{*} f\right)(x)-g(x)\right|^{2}}{\sigma^{2}}\right\} d x \\
& +\frac{1}{2} \int_{\Omega} \ln \left(\sigma^{2}\right) d x
\end{aligned}
$$

where $\sigma^{2}$ is an unknown constant.

Minimizing the above equation is equivalent to minimizing the residual $[19,20]$

$$
\frac{1}{2}\|k * f-g\|_{L^{2}}^{2}
$$

The minimization problem defined above is ill-posed, hence we incorporate a regularization term and gets the following cost functional [17-18]

$$
E\left(f, \sigma^{2}\right)=E_{1}\left(f, \sigma^{2}\right)+\lambda J(f)
$$

Considering TV regularization term as

$$
J(f)=\int_{\Omega} \sqrt{|\nabla f|^{2}+\beta} d x
$$

$$
\begin{aligned}
& E_{1}\left(f, \sigma^{2}\right)=\frac{1}{2} \int_{\Omega}\left\{\frac{\left|\left(k^{*} f\right)(x)-g(x)\right|^{2}}{\sigma^{2}}\right\} d x \\
& +\frac{1}{2} \int_{\Omega} \ln \left(\sigma^{2}\right) d x+\lambda \int_{\Omega} \sqrt{|\nabla f|^{2}+\beta} d x
\end{aligned}
$$

\section{Algorithm:}

Choose initial values of $f^{0}$ and $\left(\sigma^{2}\right)^{0}$. For different values of $n=1,2,3,4, \ldots \ldots$ so on

1. Evaluate $f^{n+1}$, under the condition

$$
f^{n+1}=\arg \min E\left(f,\left(\sigma^{2}\right)^{n}\right)
$$


2. Evaluate $\left(\sigma^{2}\right)^{n+1}$, under the condition

$$
\left(\sigma^{2}\right)^{n+1}=\arg \min E\left(f^{n+1},\left(\sigma^{2}\right)\right)
$$

Check for the convergence, if converges STOP, else go to STEP 1.

\section{Gaussian MiXture-Total Variation Model}

(GM-TV MODEL)

Assume at each point $x \in \Omega$, the intensity of noise $n(x)$ or $(k * f)(x)-g(x)$ is a random variable and all the random variables $\{n(x) \mid x \in \Omega\}$ are independent and identically-distributed with the following probability density function [19]:

$$
p(n(x) \mid \Theta)=\sum_{l=1}^{M} \alpha_{l} p_{l}\left(n(x) \mid \mu_{l}, \sigma_{l}^{2}\right)
$$

where each $p_{l}$ is a Gaussian density function with mean $\mu_{l}$ and variance $\sigma_{l}^{2}$, and the parameter set $\Theta=\left\{\alpha_{1}, \ldots, \alpha_{M}, \mu_{1}, \ldots, \mu_{M}, \sigma_{1}^{2}, \ldots, \sigma_{M}^{2}\right\} \quad$ is chosen such that

$$
\sum_{l=1}^{M} \alpha_{l}=1
$$

In other words, the probability density function (PDF) is a mixture of $M$ individual Gaussian components with different ratios.

$$
\begin{gathered}
E_{1}(f, \Theta)=\int_{\Omega}-\ln \sum_{l=1}^{M} \frac{\alpha_{l}}{\sigma_{l}} \exp \left\{-\frac{\left|(k * f)(x)-g(x)-\mu_{l}^{2}\right|^{2}}{2 \sigma_{l}^{2}}\right\} d x \\
J(f)=J_{\beta}(f)=\int_{\Omega} \sqrt{|\nabla f|^{2}+\beta} d x
\end{gathered}
$$

The above is to be minimized under the constraints $\sum_{l=1}^{M} \alpha_{l}=1$. Thus the problem is very much similar to the Gauss model except a new constraint.

\section{Algorithm 1:}

1. Evaluate $f^{n+1}$, under the condition

$$
f^{n+1}=\arg \min E\left(f,(\Theta)^{n}\right)
$$

2. Evaluate $(\Theta)^{n+1}$, under the condition

$$
(\Theta)^{n+1}=\arg \min E\left(f^{n+1},(\Theta)\right)
$$

3. Check for the convergence, if converges STOP, else go to STEP 1.

In step 2 , it is noticeable that parameters of mixed pdf need to be evaluated. For minimization, we modify function as

$$
\begin{aligned}
\hat{E}_{1}(f, \Theta) & =\frac{1}{2} \sum_{l=1}^{M} \int_{\Omega}\left\{\frac{\left|\left(k^{*} f\right)(x)-g(x)-\mu_{l}^{2}\right|^{2}}{2 \sigma_{l}^{2}}\right\} \omega_{l}^{n}(x) d x \\
& +\frac{1}{2} \sum_{l=1}^{M} \int_{\Omega} \ln \left(\sigma_{l}^{n}\right) \omega_{l}^{n}(x) d x \\
& -\sum_{l=1}^{M} \int_{\Omega} \ln \left(\alpha_{l}\right) \omega_{l}^{n}(x) d x+\sum_{l=1}^{M} \int_{\Omega} \alpha_{l} d x
\end{aligned}
$$

Where

$$
\begin{gathered}
\omega_{l}^{n}(x)=\omega_{l}\left(x \mid\left(k * f^{n}\right)(x)-g(x), \Theta^{n}\right) \\
\omega_{l}^{n}(x)=\frac{\alpha_{l}^{n} p_{l}\left(k * f^{n}\right)(x)-g(x) \mid \mu_{l}^{n},\left(\sigma_{l}^{2}\right)^{n}}{\sum_{v=1}^{M} \alpha_{v}^{n} p_{v}\left(k * f^{n}\right)(x)-g(x) \mid \mu_{v}^{n},\left(\sigma_{v}^{2}\right)^{n}}
\end{gathered}
$$

After adding variation parameter we get,

$$
\begin{aligned}
& \hat{E}_{1}(f, \Theta)=\frac{1}{2} \sum_{l=1}^{M} \int_{\Omega}\left\{\frac{\left|\left(k^{*} f\right)(x)-g(x)-\mu_{l}^{2}\right|^{2}}{2 \sigma_{l}^{2}}\right\} \omega_{l}^{n}(x) d x \\
& \quad+\frac{1}{2} \sum_{l=1}^{M} \int_{\Omega} \ln \left(\sigma_{l}^{n}\right) \omega_{l}^{n}(x) d x- \\
& \sum_{l=1}^{M} \int_{\Omega} \ln \left(\alpha_{l}\right) \omega_{l}^{n}(x) d x+\sum_{l=1}^{M} \int_{\Omega} \alpha_{l} d x+\int_{\Omega} \sqrt{|\nabla f|^{2}+\beta} d x
\end{aligned}
$$

Algorithm 2:

Choose initial values, $f^{0}, \Theta^{0}$ and calculate $\omega_{l}^{0}(x)$. For different values of $n=1,2,3, \ldots$.

Find $f^{n+1}$, such that

$$
\frac{f^{n+1}-f^{n}}{\Delta t}=\lambda\left(\frac{\nabla f^{n}}{\sqrt{\left|\nabla f^{n}\right|^{2}+\beta}}\right)-\sum_{l=1}^{M} \frac{\hat{k}\left(k * f^{n}-g-\mu_{l}^{n}\right) \omega_{l}^{n}}{\left(\sigma^{2}\right)^{n}}
$$

Find, $\Theta^{n+1}$ and $\omega_{l}^{n+1}$ such that 


$$
\begin{gathered}
\alpha_{l}^{n+1}=\frac{1}{A} \int_{\Omega} \omega_{l}^{n}(x) d x \\
A=\int_{\Omega} 1 d x \\
\mu_{l}^{n+1}=\frac{\int_{\Omega}\left|\left(k * f^{n+1}\right)(x)-g(x)\right| \omega_{l}^{n} d x}{\int_{\Omega} \omega_{l}^{n} d x} \\
\left(\sigma_{l}^{2}\right)^{n+1}=\frac{\int_{\Omega}\left|\left(k * f^{n+1}\right)(x)-g(x)-\mu_{l}^{n+1}\right|^{2} \omega_{l}^{n} d x}{\int_{\Omega} \omega_{l}^{n} d x}(24) \\
\omega_{l}^{n+1}(x)=\frac{\alpha_{l}^{n+1} p_{l}\left(k * f^{n+1}\right)(x)-g(x) \mid \mu_{l}^{n+1},\left(\sigma_{l}^{2}\right)^{n+1}}{\sum_{v=1}^{M} \alpha_{v}^{n+1} p_{v}\left(k * f^{n+1}\right)(x)-g(x) \mid \mu_{v}^{n+1},\left(\sigma_{v}^{2}\right)^{n+1}} \\
p_{l}=\left(\left(k * f^{n+1}\right)(x)-g(x) \mid \mu_{l}^{n+1},\left(\sigma_{l}^{2}\right)^{n+1}\right) \\
p_{l}=\frac{1}{\sqrt{2 \pi\left(\sigma_{l}^{2}\right)^{n+1}}} \operatorname{exp-}\left(\frac{\left|\left(k * f^{n+1}\right)(x)-g(x)-\mu_{l}^{n+1}\right|^{2}}{2\left(\sigma_{l}^{2}\right)^{n+1}}\right)
\end{gathered}
$$

This is known as Gaussian Mixture-Total Variation equation. The key point in this model is the introduction of $\omega_{l}^{n}(x)$ which lies between 0 and 1. For each point $x \in \Omega, \sum_{l=1}^{M} \omega_{l}^{n}(x)=1$. The $M=1$ values of $\omega_{l}^{n}(x)$ can be thought as weighing average of $M$ deblurring components. Here, if $M=1$, this model is equivalent to Gauss model.

\section{RESULTS}

In this section, simulation results for both Gauss and Gaussian Mixture model are presented. In Fig. 3, Lenna image is shown which is made blur using Gaussian blur with variance 1 . However, no noise is added to the original image. Thus, these results can be thought of as de-blurring results. The recovered images using G-TV and GM-TV are shown in Fig. 3(c) and (d) respectively. It is found that the image is well recovered after 271 iterations. Moreover, the quality of the recovered image is good.
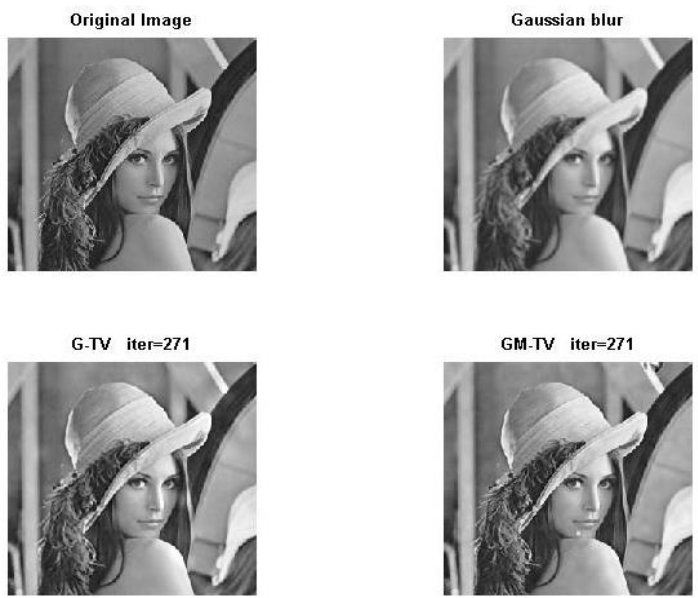

Fig.3. (a) Original image (b) Gaussian blur with variance 1 (c) G-TV iteration 271 (d) GM-TV iteration 271

The obtained PSNR and SSIM from G-TV model are $44 \mathrm{~dB}$ and 0.912 and from GM-TV model obtained PSNR and SSIM, are $44 \mathrm{~dB}$ and 0.92 . Thus the performance of the GM-TV model is superior to G-TV model in case of de-blurring of images.

In Fig. 4, estimated pdfs are shown. As noise is not present therefore a vertical line is observed. However, due to blurring pixels values change slightly, thus a smaller broadening is observed in estimated pdf. The broadening in estimated pdf using GM-TV model is lesser in comparison to G-TV model.

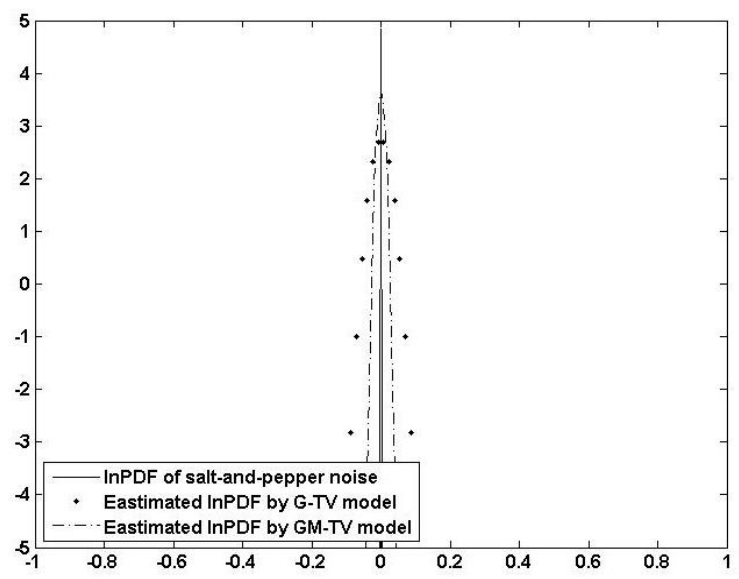

Fig.4. Estimated PDF by both the models (var=1)

In Fig. 5, Lenna image is shown which is made blur using Gaussian blur with variance 9. The recovered images using G-TV and GM-TV are shown in Fig. 5(c) and (d). It is found that images are well recovered after 441 iterations. Moreover, the quality of the recovered image is again good. But the performance of GM-TV model is much better. Thus as the blurring increases more number of iterations are required to recover the image. The obtained PSNR and SSIM from G-TV model are $29.8 \mathrm{~dB}$ and 0.65 and from GM-TV model obtained PSNR and SSIM are 39.2 and 0.80 . Thus the performance of the GM-TV model is much superior to G-TV model in case of de-blurring of images. 

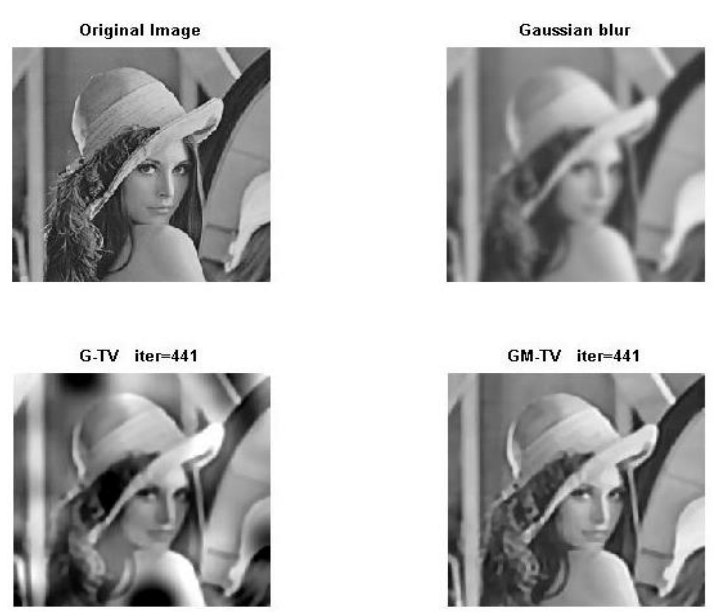

Fig.5. Original image (b) Gaussian blur with variance 9 (c) G-TV iteration 441 (d) GM-TV iteration 441

In Fig. 6, estimated pdfs are shown. As noise is not present therefore a vertical line is observed. However, due to moderate blurring pixels value changes, thus a broadening is observed in estimated pdf. The broadening in estimated pdf using GM-TV model is lesser in comparison to G-TV model. It can be inferred from the figure that as the variance increases the obtained pdf broadening in G-TV model is more. Thus, G-TV model is more susceptible to blurring in comparison to GM-TV model.

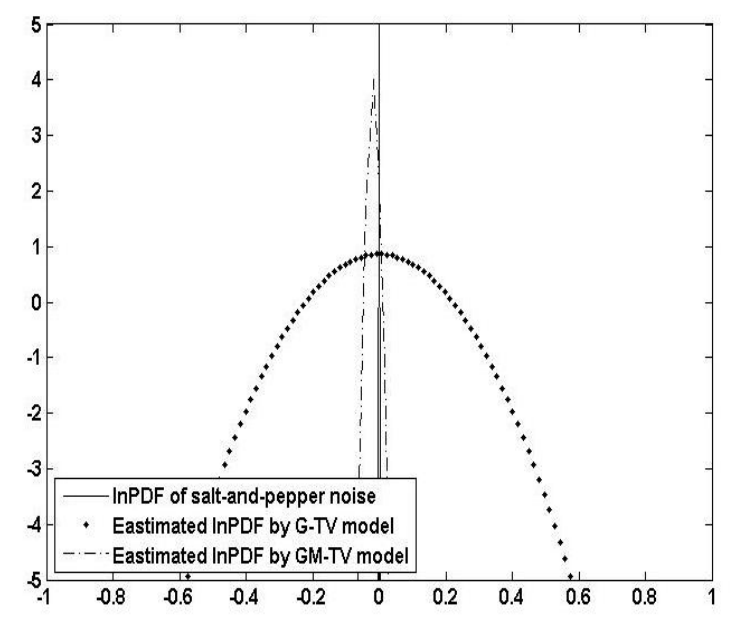

Fig.6. Estimated PDF by both the models (var=9)

In Fig. 7, Lenna image is shown which is made blurred using Gaussian blur with variance 25. The recovered images using G-TV and GM-TV are shown. It is found that images are not well recovered after 51 iterations. As we increase the number of iterations due to the over fitting of Gaussian model recovered image becomes black. Therefore is case of heavily blurred image quality of recovered image is not good. The obtained PSNR and SSIM from G-TV model are $12.28 \mathrm{~dB}$ and 0.54 and from GM-TV model obtained PSNR and SSIM are $13.05 \mathrm{~dB}$ and 0.66 .
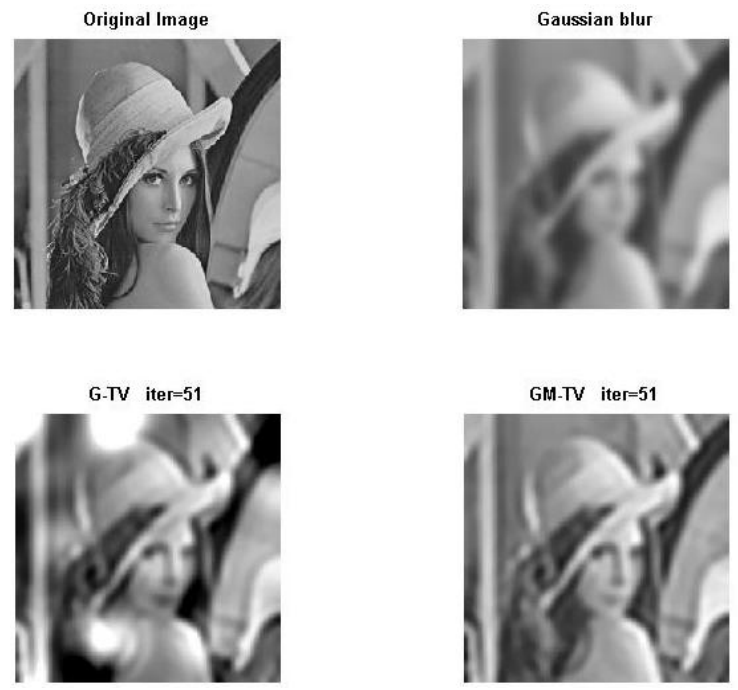

Fig.7. (a) Original image (b) Gaussian blur with variance 25 (c) G-TV iteration 441 (d) GM-TV iteration

In Fig. 8, estimated pdfs are shown. As noise is not present therefore a vertical line is again observed. However, due to high blurring pixels value changes significantly, thus a larger broadening is observed in estimated pdf. The broadening in estimated pdf using GM-TV model is lesser in comparison to G-TV model. From Fig .4, 6 and 8, it can be observed that estimated pdf from GM-TV model remains nearly same while for G-TV model it broadens as blurring increases.

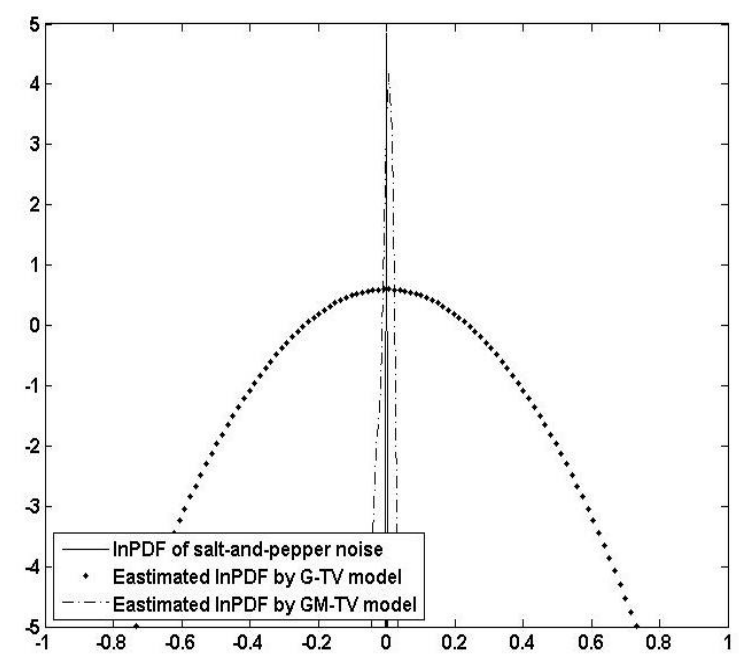

Fig.8. Estimated PDF by both the models (var=25)

In Fig. 9, PSNR (dB) vs. sigma is plotted in the form of BAR graph. It is clear from the figure that as the variance increases, the value of PSNR decreases. 


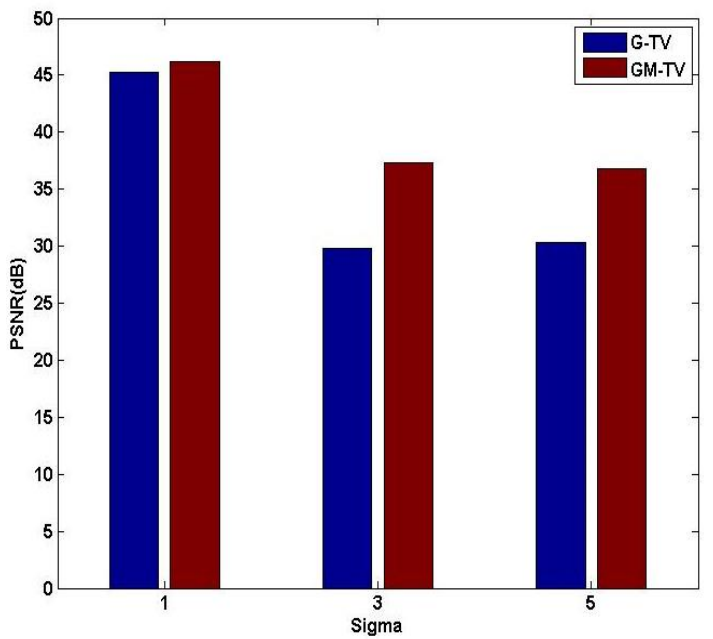

Fig.9. PSNR (dB) vs. Sigma (blur only)

Similarly, SSIM vs. sigma is plotted in Fig. 10. Here SSIM decreases for both the methods as sigma increases. However for sigma 3 and 5, PSNR is nearly same (Fig. 9), in fact it is more for sigma 3 . Thus, PSNR cannot be considered as good quality measure.

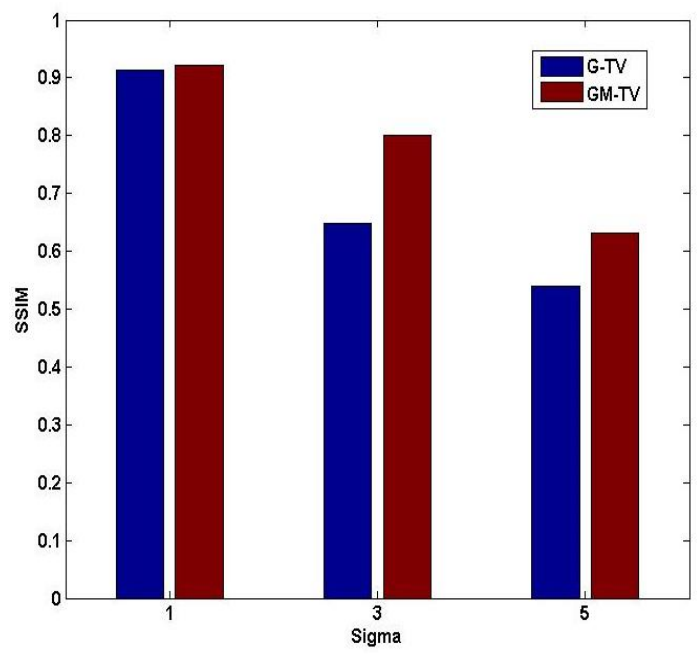

Fig.10 SSIM vs. Sigma (blur only)

In Fig. 11, only Gaussian blurring is considered with mean 25 and variance 1 . Thus, due to the lesser variance blurring is less. In Fig. 11, the Gaussian blurred image is very much similar to the original Lenna image however as noise is introduced, the blurred and noisy image is not clearly visible. The recovered images using G-TV and GM-TV are shown, and it is clear that the recovery of the image is better in case of GM-TV model. Still recovered images are not of good quality especially under G-TV model.
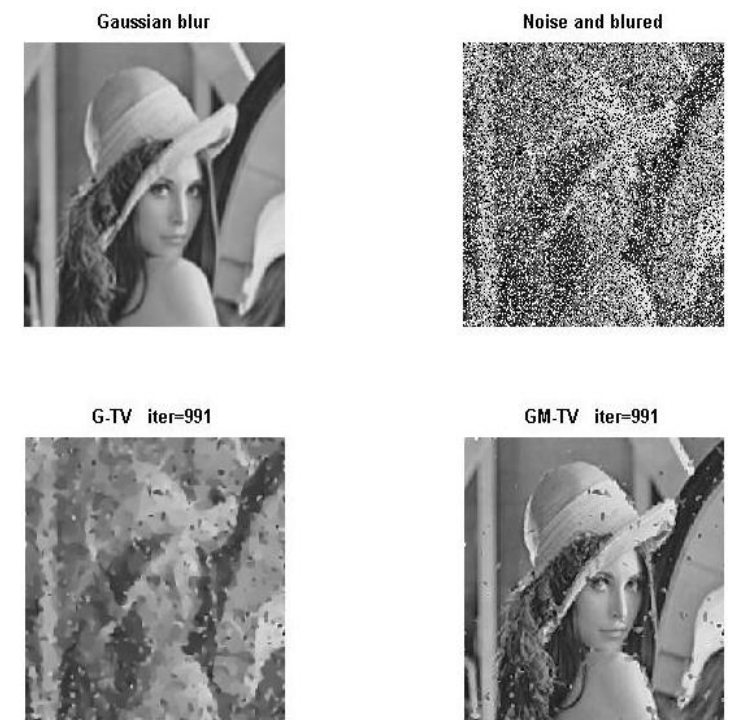

Fig.11. (a) Gaussian blur (b) Gaussian and salt and pepper noise with variance 1 (c) G-TV iteration 991 (d) GM-TV iteration 991

In Fig. 12, obtained pdfs for G-TV and GM-TV are shown. Now due to presence of salt and pepper noise a random pdf which is somewhat similar about vertical axis is shown. In presence of noise estimated pdf by G-TV model is broader in comparison to earlier case of blur. A major change is observed in GM-TV model pdfs, it is very broad now.

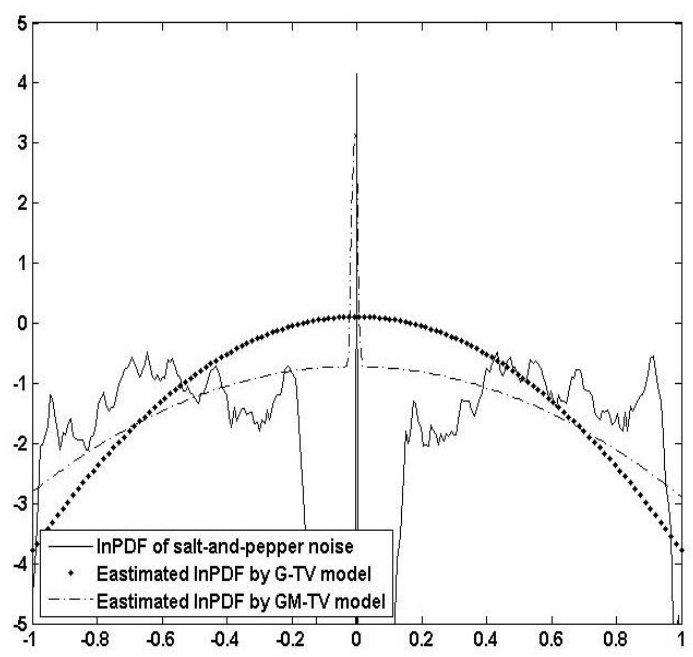

Fig.12 Estimated PDF by both the models in presence of noise (var=1)

In Fig. 13, only Gaussian blurring is considered with mean 25 and variance 9. Thus due to lesser variance, blurring is less. The recovered images using G-TV and GM-TV are shown, and it is clear that the recovery of image is better in case of GM-TV model. Still recovered image under G-TV model is of poor quality. Now due to larger blurring, pixel expansion is much and GM-TV model is now able to distinguish between blur and noise, thus results improve. 

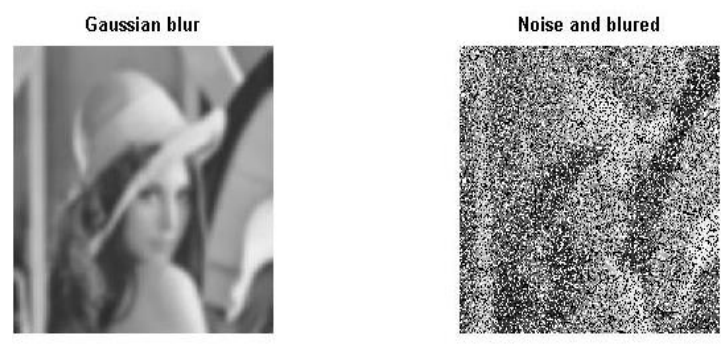

G-TV iter=1991
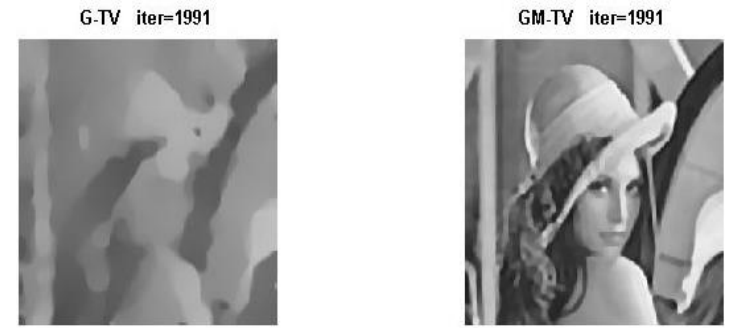

Fig.13. (a) Gaussian blur (b) Gaussian and salt and pepper noise with variance 9 (c) G-TV iteration 1991 (d) GM-TV iteration 1991

In Fig. 14, obtained pdfs for G-TV and GM-TV are shown. Now due to presence of salt and pepper noise, a random pdf which is somewhat similar about vertical axis is shown. In presence of noise estimated pdf by G-TV model is broader in comparison to earlier case of blur. A major change is observed in GM-TV model pdfs, it is very broad now. However, with increase in variance no significant changes are observed in the graphs.

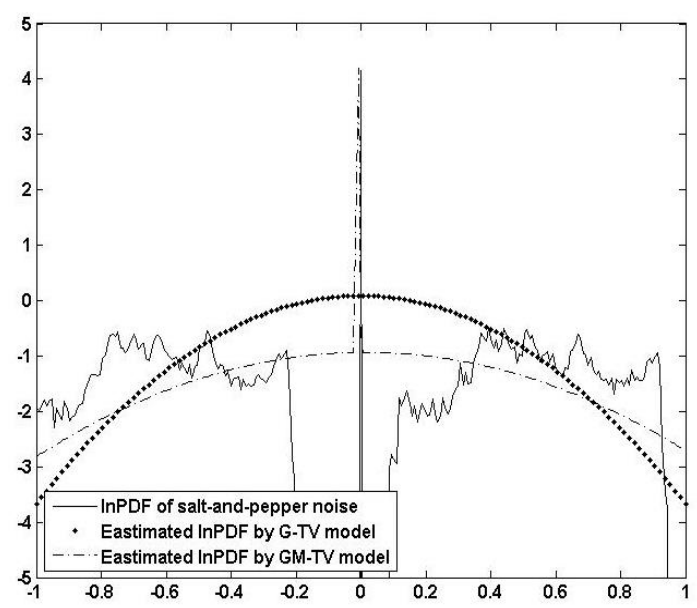

Fig.14. Estimated PDF by both the models in presence of noise (var=9)

In Fig. 15, only Gaussian blurring is considered with mean 25 and variance 25 . Thus due to the lesser variance blurring is less. The recovered images using G-TV and GM-TV are shown, and it is clear that the recovery of image is better in case of GM-TV model. Still recovered image under G-TV model is of poor quality. Although the performance of GM-TV is not good, yet it is better than G-TV model.
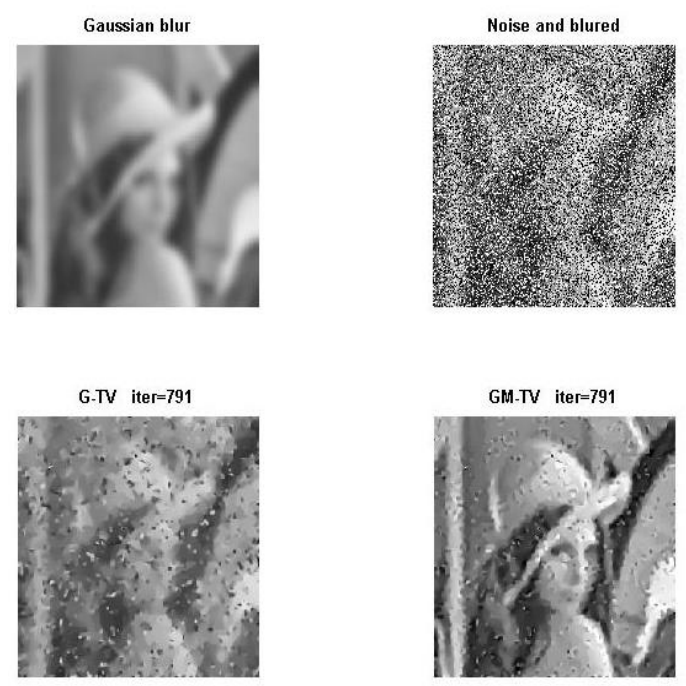

Fig.15. (a) Gaussian blur (b) Gaussian and salt and pepper noise with variance 25 (c) G-TV iteration 791 (d) GM-TV iteration 791

In Fig. 16, obtained pdfs for G-TV and GM-TV are shown. Now due to presence of salt and pepper noise a random pdf which is somewhat similar about vertical axis is shown. In presence of noise estimated pdf by G-TV model is broader in comparison to earlier case of blur. A major change is observed in GM-TV model pdfs, it is very broad now. However, with increase in variance no significant changes are observed in the graphs (Fig. 14 and Fig. 16).

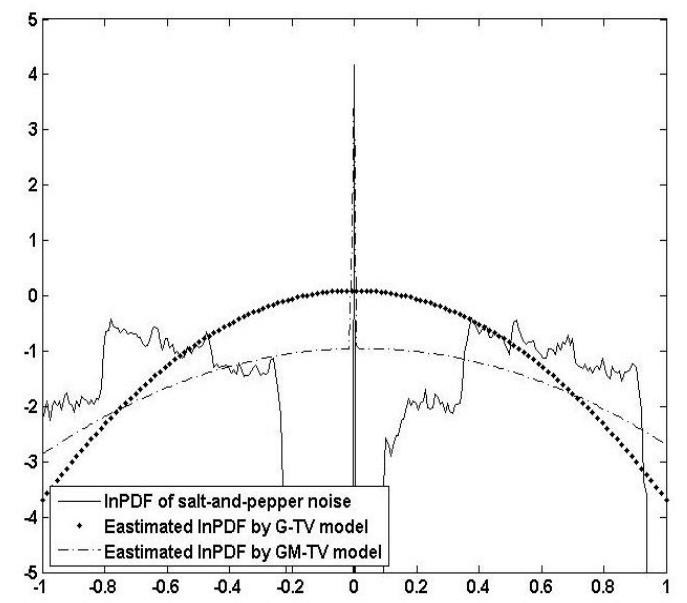

Fig.16. Estimated PDF by both the models in presence of noise (var=25)

In Fig. 17, PSNR (dB) vs. sigma is plotted in the form of BAR graph. It is clear from the figure that as the variance increases the value of PSNR decreases. However for sigma 1 the PSNR $(\mathrm{dB})$ for G-TV model is 32.7 while for GM-TV it is 37.6. For sigma equals 3 and 5, PSNR for G-TV is 31.2 and 31.8 respectively. For GM-TV for sigma 3 and 5, PSNR for GM-TV is 38.4 and 34.3 respectively. Thus obtained values clearly suggest that GM-TV model is much more superior to G-TV model. 


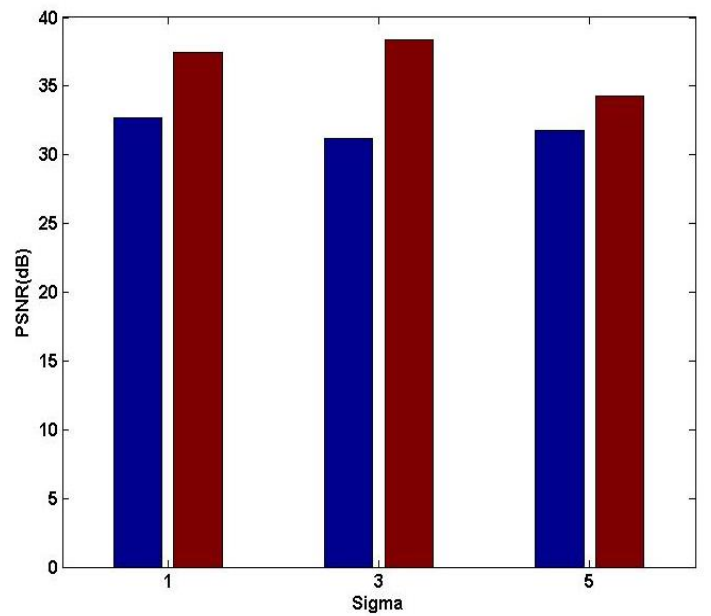

Fig.17. PSNR (dB) vs. Sigma (Blur and noise)

Similarly, SSIM vs. sigma is plotted in Fig. 18. Here SSIM decreases for both the methods as sigma increases. However for sigma 1 the SSIM for G-TV model is 0.558 while for GM-TV it is 0.802 . For sigma equals 3 and 5, SSIM for G-TV is 0.524 and 0.323 respectively. For GMTV for sigma 3 and 5, SSIM for GM-TV is 0.8 and 0.507 respectively. Thus obtained values clearly suggest that GM-TV model is much more superior to G-TV model.

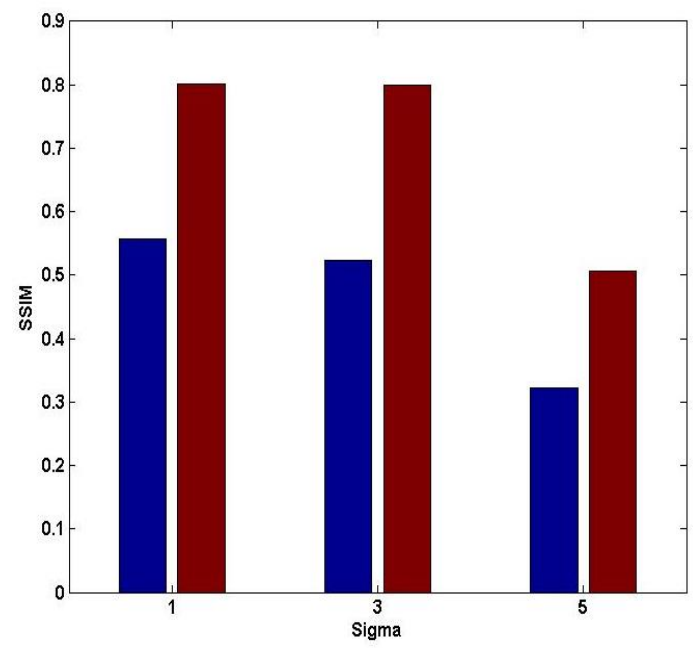

Fig.18. SSIM vs. Sigma (Blur and noise)

\section{CONCLUSIONS}

In this paper, two methods are detailed which are capable of removing blurring and noises in digital images.

On the basis of obtained results, following conclusions can be made:

- G-TV model is quite effective in reconstructing images with blur and uniform distributed noise without changing the regularization parameter $\lambda$ directly.

- However, it still could not work well when the image is contaminated with blur and mixed noise.
- Moreover, with lesser Gaussian blur variance, image recovered in lesser iterations.

- However, as the variance increases, number of iterations required to recover images also increases.

- G-TV model fails to remove noise; however, it performs well for de-blurring of the image.

- GM-TV model works well even on noisy images.

- GM-TV model is also capable of removing both blurring and noise in the images.

\section{REFERENCES}

[1] Bilmes, Jeff A. "A gentle tutorial of the EM algorithm and its application to parameter estimation for Gaussian mixture and hidden Markov models." International Computer Science Institute 4.510 (1998): 126.

[2] Acar, Robert, and Curtis R. Vogel. "Analysis of bounded variation penalty methods for ill-posed problems." Inverse problems 10.6 (1994): 1217.

[3] Rudin, Leonid I., and Stanley Osher. "Total variation based image restoration with free local constraints." Image Processing, 1994. Proceedings. ICIP-94., IEEE International Conference. Vol. 1. IEEE, 1994.

[4] Vogel, Curtis R., and Mary E. Oman. "Fast, robust total variation-based reconstruction of noisy, blurred images." IEEE transactions on image processing 7.6 (1998): 813824.

[5] Redner, Richard A., and Homer F. Walker. "Mixture densities, maximum likelihood and the EM algorithm." SIAM review 26.2 (1984): 195-239.

[6] Chan, Raymond H., Chung-Wa Ho, and Mila Nikolova. "Salt-and-pepper noise removal by median-type noise detectors and detail-preserving regularization." IEEE Transactions on image processing 14.10 (2005): 14791485.

[7] Chan, Tony F., and Chiu-Kwong Wong. "Total variation blind deconvolution." IEEE transactions on Image Processing 7.3 (1998): 370-375.

[8] Nikolova, Mila. "A variational approach to remove outliers and impulse noise." Journal of Mathematical Imaging and Vision 20.1 (2004): 99-120.

[9] Bar, Leah, Nahum Kiryati, and Nir Sochen. "Image deblurring in the presence of impulsive noise." International Journal of Computer Vision 70.3 (2006): 279-298.

[10] Shi, Yuying, and Qianshun Chang. "Acceleration methods for image restoration problem with different boundary conditions." Applied Numerical Mathematics 58.5 (2008): 602-614.

[11] Bect, Julien, et al. "A | 1-unified variational framework for image restoration." Computer Vision-ECCV 2004 (2004): 1-13.

[12] Shinde, Bhausaheb, Dnyandeo Mhaske, and A. R. Dani. "Study of noise Detection and noise removal techniques in medical images." International Journal of Image, Graphics and Signal Processing 4.2 (2012): 51.

[13] Bandyopadhyay, Aritra, et al. "A relook and renovation over state-of-art salt and pepper noise removal techniques." International Journal of Image, Graphics and Signal Processing 7.9 (2015): 61.

[14] Mahmoud, Amira A., et al. "Comparative study of different denoising filters for speckle noise reduction in ultrasonic b-mode images." International Journal of Image, Graphics and Signal Processing 5.2 (2013): 1.

[15] He, Lin, Antonio Marquina, and Stanley J. Osher. "Blind deconvolution using TV regularization and Bregman 
iteration." International Journal of Imaging Systems and Technology 15.1 (2005): 74-83.

[16] Shi, Yuying, and Qianshun Chang. "New time dependent model for image restoration." Applied mathematics and computation 179.1 (2006): 121-134.

[17] Rudin, Leonid I., Stanley Osher, and Emad Fatemi. "Nonlinear total variation based noise removal algorithms." Physica D: Nonlinear Phenomena 60.1-4 (1992): 259-268.

[18] Lagendijk, Reginald L., Jan Biemond, and Dick E. Boekee. "Regularized iterative image restoration with ringing reduction." IEEE Transactions on Acoustics, Speech, and Signal Processing 36.12 (1988): 1874-1888.

[19] L.A. Vese, Variational Methods in Image Processing, Chapman \& Hall, CRC Press, Boca Raton, FL, 2012.

[20] Wang, Y., et al. "MTV: modified total variation model for image noise removal." Electronics Letters 47.10 (2011): 592-594.

\section{Authors' Profiles}

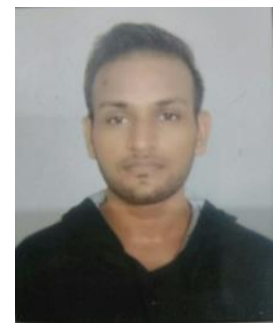

Vipul Goel was born in Baghpat district, Uttar Pradesh, India. He has completed his Bachelor of Technology degree in Electronics and Communication Engineering from Aryabhatt College affiliated to Uttar Pradesh Technical University, Uttar Pradesh, India. He is currently pursuing his Master of Technology degree in Electronics and Communication from Harcourt Butler Technical University, Kanpur, Uttar Pradesh, India. His research interests include image and video processing, machine learning and computer vision.

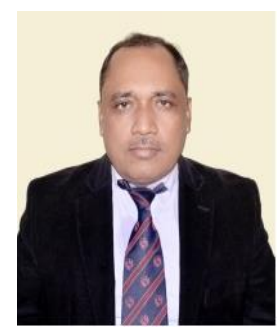

Dr. Krishna Raj did his Bachelor's degree in Electronics Engineering from M.M.M.E.C., Gorakhpur in 1992 and pursued his Master's degree in Electronics Engineering with Control \& Instrumentation as specialization from M.N.N.I.T., Allahabad (formerly M.N.R.E.C.), Uttar Pradesh, India in 1995. He has completed his doctoral degree in Electronics Engineering from M.M.M.E.C. Gorakhpur D.D.U. University of Gorakhpur, Uttar Pradesh State, in 2003.

His field of interests includes digital signal processing, image processing, computer arithmetic, and VLSI. Currently, he is working as a professor in Electronic Engineering department of Harcourt Butler Technical University, Kanpur, Uttar Pradesh state. He has over 22 years of teaching and research experience. He has published more than 42 technical papers in national and international conferences and more than 20 articles/ research papers in journals. He has authored 3 books in the field of signal processing and digital logic design.

Dr. K. Raj has the fellowship of IETE and membership of IEEE. He is also a life member of IE.

How to cite this paper: Vipul Goel, Krishna Raj," Removal of Image Blurring and Mix Noises Using Gaussian Mixture and Variation Models", International Journal of Image, Graphics and Signal Processing(IJIGSP), Vol.10, No.1, pp. 47-55, 2018.DOI: $10.5815 /$ ijigsp.2018.01.06 